Military Technical College

Kobry El-Kobbah, Cairo, Egypt

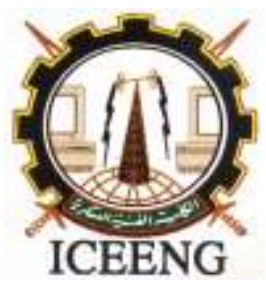

\section{$11^{\text {th }}$ International Conference on Electrical Engineering ICEENG 2018}

\title{
A comparative study on Turn Model Routing Algorithms Applied to Mesh Topology Network-on-Chip
}

\author{
Sherif Heshmat*, Tarek Mahmoud*, and Mahmoud Safwat*
}

\section{Abstract:}

The enormous increase in the number of transistors on the same chip and the increase of the size of system itself, lead to many challenges to the implementation of system on chip (SoC). These challenges are solved using the network on chip (NoC) methodology. NoC routing is one of the most important challenges that affect the whole performance of the system. Routing can affect the latency of the network as well as the entire throughput of the network. In this paper, a comparative study is performed on XY routing algorithm (deterministic routing algorithm), fully adaptive XY routing algorithm (fully adaptive routing algorithm) and two partially adaptive routing algorithms (the odd even $(\mathrm{OE})$ routing algorithm and the abacus turn model (AbTM) routing algorithm) on four traffic patterns. Booksim simulator is used in this work. The AbTM proved to be the best routing algorithm for the performance of the NoC in terms of latency and throughput. The AbTM settles the network to maximum even adaptiveness among all its routers.

\section{Keywords:}

Network on chip; XY routing algorithm; Odd even routing algorithm; Abacus turn model routing algorithm 


\section{Introduction:}

With the fast evolution of very large scale integrated circuits (VLSI) technologies and the enormous increase in number of transistors on integrated circuits a new paradigm appeared to the top which is the SoC. Because the growth of VLSI and the terminals connected to $\mathrm{SoC}$ is in continuous increase, a new paradigm appeared again to solve the issues $\mathrm{SoC}$ is not able to solve. This new technology is NoC [1-4].

$\mathrm{NoC}$ is a new paradigm where a programmable system transports data between its terminals via routers. These terminals are called intellectual properties (IP) and these routers have many types of switching circuits that determine how the network will delivers its packets according to the routing algorithm which will be investigated later in this paper.

There are many parameters that affect the performance of NoC such as the network topology, routing methodology, router micro-architecture, power consumption and flow control [5]. Hence it is very important to understand the parameters that affect the NoC behavior and performance first before deciding which NoC configuration can be used with the desired system.

It was proved by Duato [6] that to avoid deadlock and livelock inside the NoC buffers must be included inside switching between a router and another. A message transporting through routers can be divided into packets. There are many packet switching techniques that can be used in NoC [7]. The first technique is store-and-forward technique. It is a simple technique, where the whole message must be received at each router before moving to another router. The store-and-forward needs a large amount of buffers and impractical for large networks. The second technique is the virtual cut-through (VCT) technique. Virtual cut-through mechanism [7] was proposed to address the large network latency problem in the store-and-forward strategy by reducing the packet delays at each routing stage. In this approach, one packet can be forwarded to the next stage before it is entirely received by the current router which reduces the store and forward delays. However, when the next stage router is not available, similar to the store-and-forward mechanism, the VCT approach also requires a large buffering space at each router to store the whole packet. The last technique is the wormhole switching technique, in this mechanism packets can be divided into smaller units called flits. Wormhole does not require the whole packet to be stored in router or must transport in certain order so it solved two main problem in the previous techniques and its transport concept depends on flit level not packet level.

Due to the complication of the VCT switching which leads to higher costs and more complexity and due to the less efficiency of store-and-forward technology, the wormhole switching technique [8] has been chosen as a packet switching mechanism in this paper. Topology of NoC defines how the routers and IPs are connected. There are a lot of topologies like ring, mesh, torus, ring etc. [5]. In this paper NoC mesh topology is used 
with dimension $4 \times 4$. The mesh topology is a general purpose architecture and suitable for many systems.

There are many simulators for NoC which simulates the entire network environment such as Noxim, RS2, OpenSoc, and Booksim. Booksim 2.0 [9] is used in this paper because it is detailed and flexible cycle-accurate NoC simulator that simulates various types of networks in terms of routing algorithms, network topologies, and router microarchitectures with different flow control that all can be modified [10].

The remaining part of the paper is organized in the following way: section II provides an overview on NoC routing techniques, section III describes the turn model routing algorithms, section IV demonstrates the simulation results and a conclusion is presented in section $\mathrm{V}$.

\section{Overview on NoC routing techniques:}

In order to understand how routing takes place inside the interconnected network we must understand the differences among the various routing methodologies. Routing is the process of transporting packets between different IPs located inside the network via other routers with respect to flow control and resource usage. Thus, different techniques for NoC routing will be discussed which the routing algorithms are inherited from.

\subsection{Source and distributed routing techniques:}

The source routing is a way for NoC routing where the source takes the decisions needed for flow control and allocates resources for the transported packets. Although the source routing may be less in complexity, it is totally ineffective when the injection rate increases in the network.

On the other hand, the distributed routing is depending on both the status of each router and the network situation. Thus, the distributed routing is a more effective technique [11] which will be used in this comparative study.

\subsection{Deterministic and adaptive routing techniques:}

These are two different versatile techniques where both have many advantages and disadvantages. The deterministic routing techniques route the packets in the same path according to the criteria of the routing algorithm itself and achieving routing with minimum latency in comparison with adaptive routing algorithms. However, it does not take into consideration the network status so it will not take different decisions if there are faulty links or high congestion in some routers. 
The adaptive routing algorithms are more complex as they take into consideration the network status and have higher latency if compared with deterministic routing algorithms. On the other hand, the adaptive routing algorithms have great advantages in solving congestion problems by choosing the less congested ports and increasing the throughput of the whole interconnected network [12].

In this comparative study we are going to show a number of algorithms for both techniques to show how the NoC will respond to these algorithms in terms of the latency and throughput of the NoC for each algorithm.

\section{Turn model routing alorithms:}

The turn model routing algorithms depends on turns in routing inside the network. Duato [6] stated that deadlock and livelock can be avoided by prohibiting cycles in the routing path so a flit transporting in the network must not make a complete cycle. So by breaking some of the turns, cycles can be avoided and Duato is applied.

There are four well known turn model routing algorithms; XY, negative first, west first and north last. The XY routing algorithm is considered as the most powerful one as it prohibits four turns of the eight possible cycles while the others prohibit only two out of eight possible cycles [11]. Other turn model routing algorithms were inherited from the XY routing algorithm. In this work and based on the turn model routing methodology, a comparative study on deterministic XY routing algorithm, fully adaptive $\mathrm{XY}$ routing algorithms, Odd even (OE) routing algorithm and abacus turn model routing algorithm (AbTM) and a comparison on their performance among different traffic patterns is carried out.

\subsection{Deterministic $X Y$ routing algorithm:}

The packet in mesh topology following deterministic XY routing algorithm moves first in the X-direction then it changes its path to the y-direction regardless of the network conditions. The arrows in Figure 1 show how packets move according to the XY routing algorithm, the packet source is router no.5 (r5) and the destination is router no.15 (r15), so after the packet moves in the x-direction to router no. 6 and no.7 it then moves along the $\mathrm{y}$-direction passing through router no.11 and finally reaching its destination. Although XY routing algorithm is simple, it is not effective in most cases [11]. 


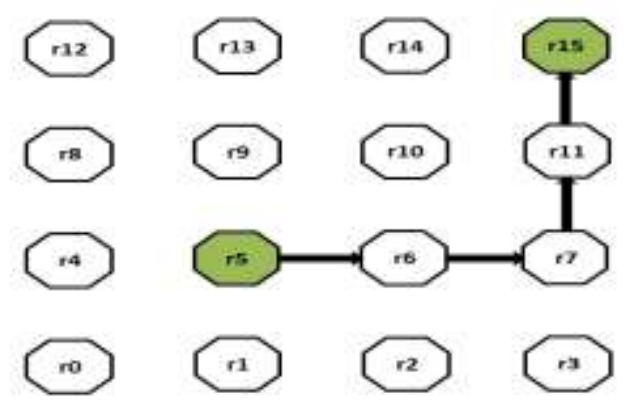

Figure (1) : Deterministic XY routing algorithm

\subsection{Fully adaptive $X Y$ routing algorithm:}

This routing algorithm is similar to the $\mathrm{XY}$ routing algorithm except that it switches between the $\mathrm{x}$-direction and y-direction in minimal routes scheme depending on the router status. The arrows in Figure 2 explain how packets move according to the fully adaptive XY routing algorithm. The less congested router is chosen rather than the more congested one. This algorithm is more complicated than the XY routing algorithm; however it gives better results in terms of the average throughput [8].

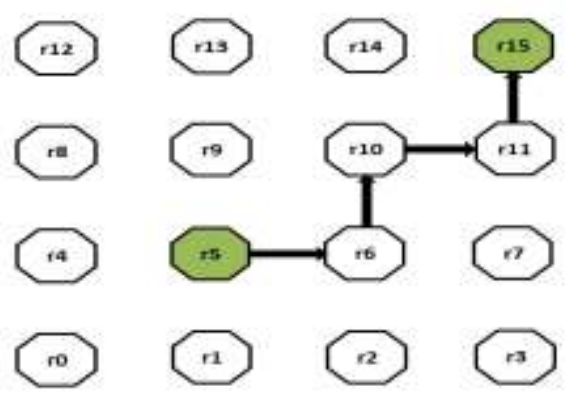

Figure (2) : Fully adaptive XY routing algorithm

$\underline{3.3 \text { Odd even }(O E) \text { routing algorithm: }}$

The $\mathrm{OE}$ routing algorithm is characterized by increasing the degree of adaptiveness of the network as compared to the fully adaptive XY routing algorithm. It is a partially adaptive routing algorithm where some constraints are placed on the movement of the packets. The rows of the mesh is divided into even and odd rows where some turns are allowed in even paths and not allowed in odd paths and vice versa [12].

Therefore, this algorithm is partially adaptive as it gives chance for adaptive choice depending on the router status and putting some constraints for others. Figure 3 demonstrates the only allowed turns in the OE routing algorithm for a $4 \times 4$ mesh topology. Although OE routing algorithm solved many issues of the deterministic XY 
routing algorithm and the fully adaptive $\mathrm{XY}$ routing algorithm, it does not solve the problem of even routing adaptiveness over the whole network.

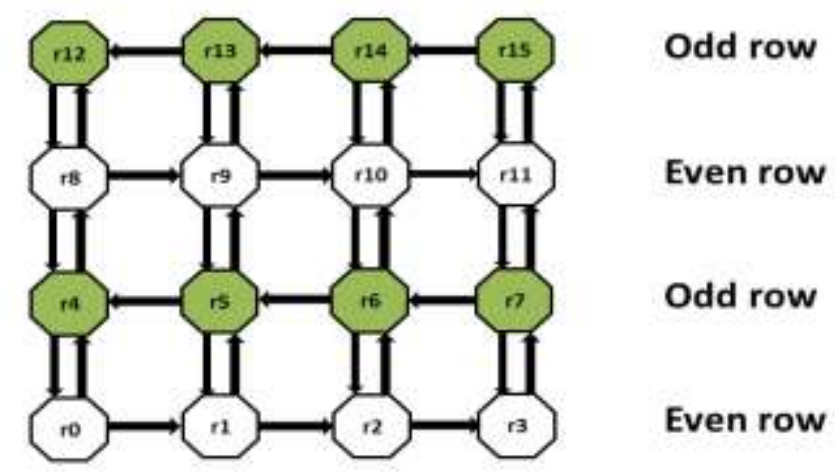

Figure (3) : OE routing algorithm

\subsection{Abacus turn model (AbTM) routing algorithm:}

The even adaptiveness of the routing algorithm across the whole network is the main disadvantage of the $\mathrm{OE}$ routing algorithm because its way for solving this problem cannot be generalized for all types of traffic applied to the network. For example if a certain router is a victim to receive high traffic of packets and other routers receive small amount of traffic, OE is not able to solve this issue. This issue is solved by the AbTM routing algorithm [13]. This algorithm gained advantages from the $\mathrm{OE}$ algorithm by dividing the mesh into even and odd rows but in AbTM, the network is separated into clockwise abacus as in Figure 4 and counter-clockwise abacus as in Figure 5. Where in each abacus the allowed and prohibited turns are determined using the location of the abacus beads. In Figure 4 the locations of the clockwise beads are at r3, r4, r10 and r14. In Figure 5 the locations of the counter-clockwise beads are r0, r7, r9, r14.

The locations of these beads are not fixed. They can be changed according to the traffic type or by using the AbTM in a reconfigurable way. In this work we investigate the AbTM routing algorithms with different traffic patterns and decide the best locations for beads to achieve best values for latency and throughput as we will see later. 


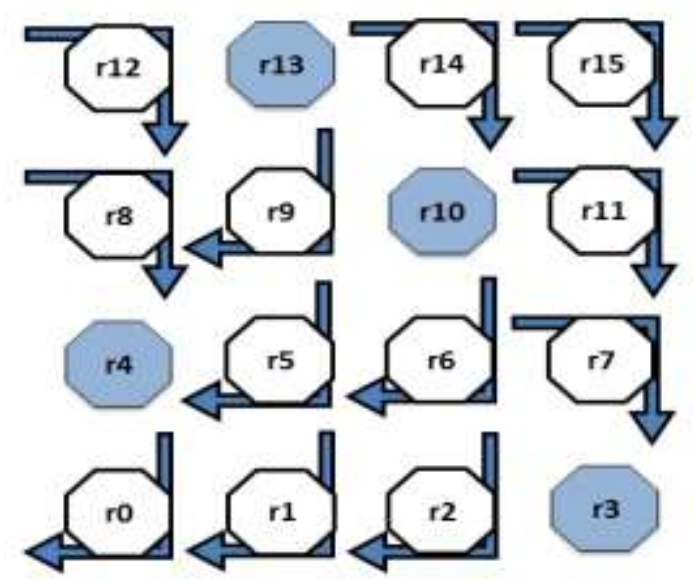

Figure (4) : AbTM routing algorithm with clockwise abacus beads

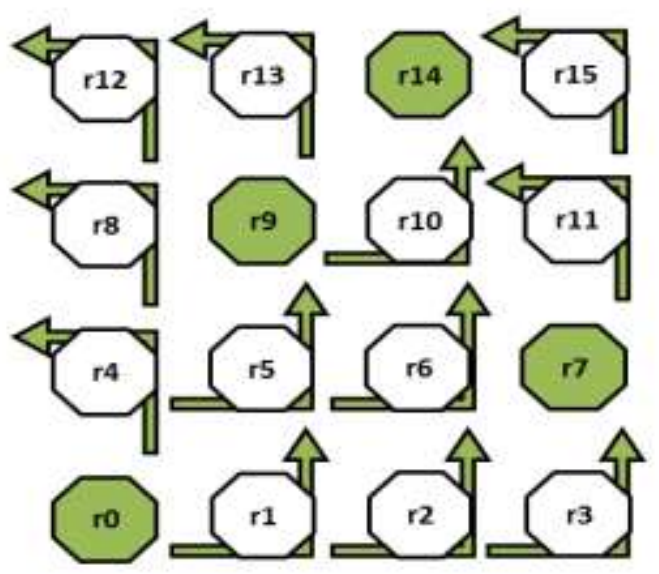

Figure (5) : AbTM routing algorithm with counter-clockwise abacus beads

\section{Simulation results:}

In this work, BookSim2.0 cycle-based network simulator [9] is used with the four tested routing algorithms developed in it. The network setting has a packet size equal to unity and virtual channels equal to eight. Furthermore, the same configurations as in [14] are applied to take advantage of comparing the achieved results using mesh topology.

Four synthetic traffic patterns, the uniform, bitrev, bitcomp, and shuffle traffic patterns [5] are applied in this comparative study. Performance metrics are average latency and average throughput for the different traffic patterns and will be shown below. AbTM is performed with a constant configuration dedicated to each traffic pattern. No results are gathered in the saturation state of the network for all routing algorithms. 
Figure 6 and Figure 7 demonstrate the latency and throughput for the routing algorithms applied to the uniform traffic pattern respectively. At the highest injection rate AbTM shows best latency, also XY and fully adaptive XY have comparable results. The throughput of AbTM is the best among all routing algorithms.

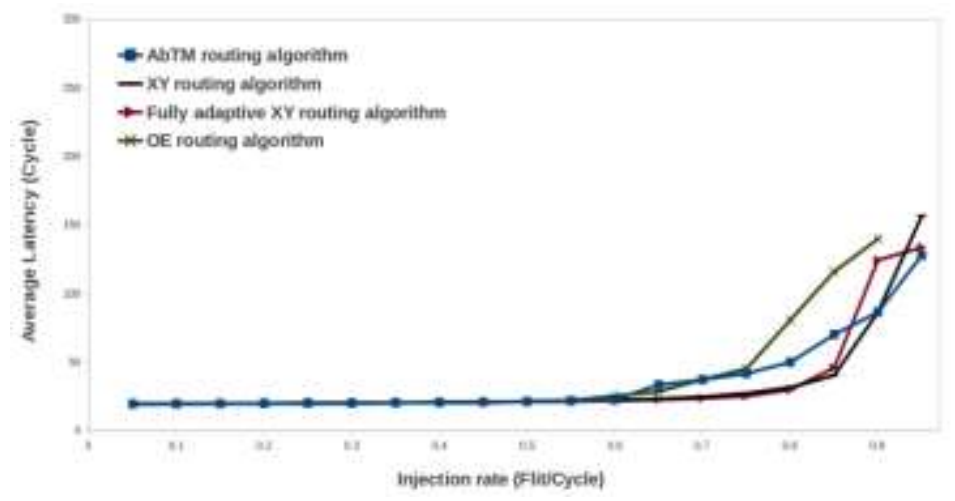

Figure (6) : Average latency of various routing algorithms on uniform traffic pattern

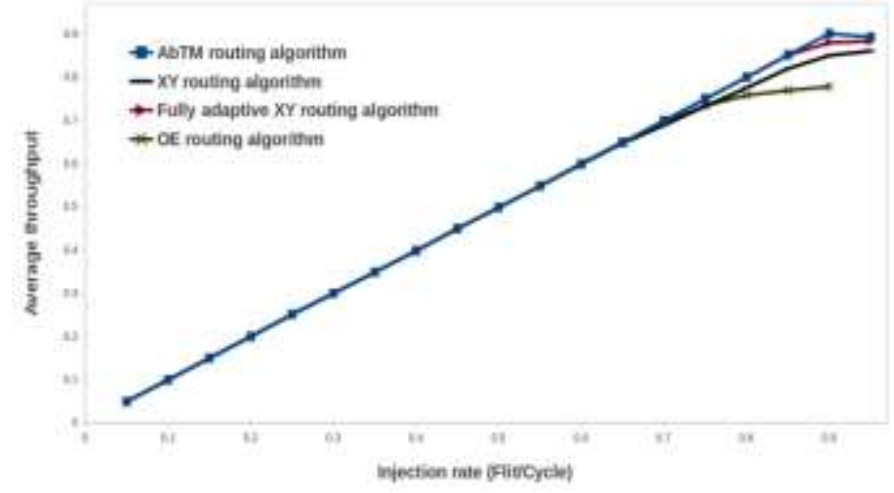

Figure (7) : Average throughput of various routing algorithms on uniform traffic pattern.

Figure 8 and Figure 9 demonstrate the latency and throughput for the routing algorithms applied to the bitrev traffic pattern respectively. AbTM shows the best latency among all algorithms. The throughput of AbTM and the fully adaptive XY routing algorithm is nearly the same with slight difference with advantage for and the throughput of the deterministic $\mathrm{XY}$ routing algorithm is the worst. 


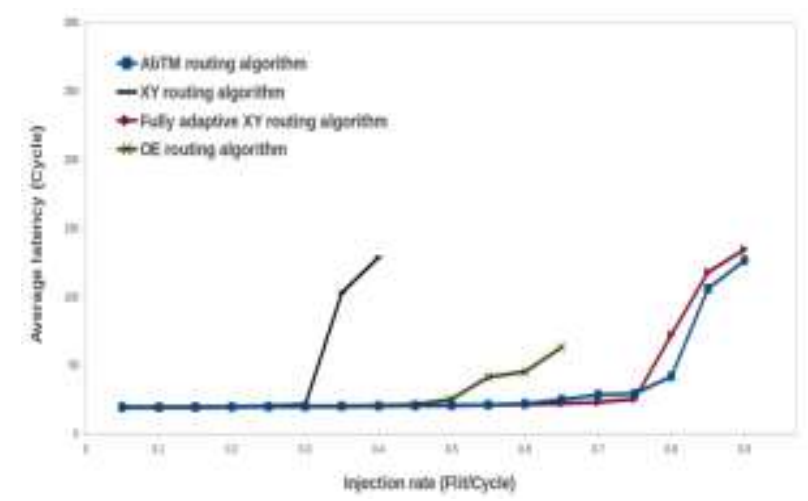

Figure (8) : Average latency of various routing algorithms on bitrev traffic pattern

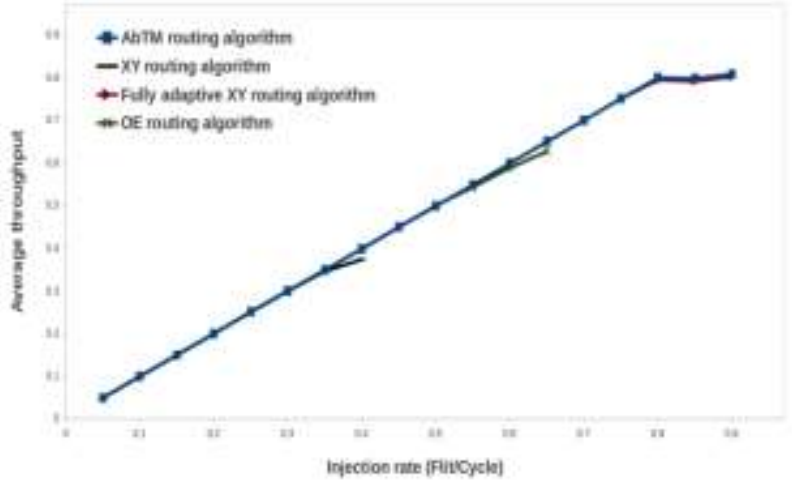

Figure (9): Average throughput of various routing algorithms on bitrev traffic pattern.

Figure 10 and Figure 11 demonstrate the latency and throughput for the routing algorithms applied to the bitcomp traffic pattern respectively. Although the AbTM does not give the best latency, it has the best throughput value among all other routing algorithms.

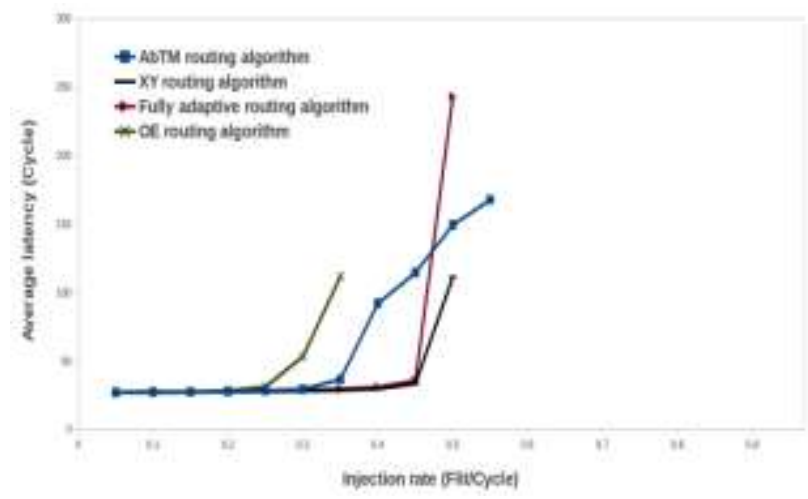

Figure (10): Average latency of various routing algorithms on bitcomp traffic pattern 


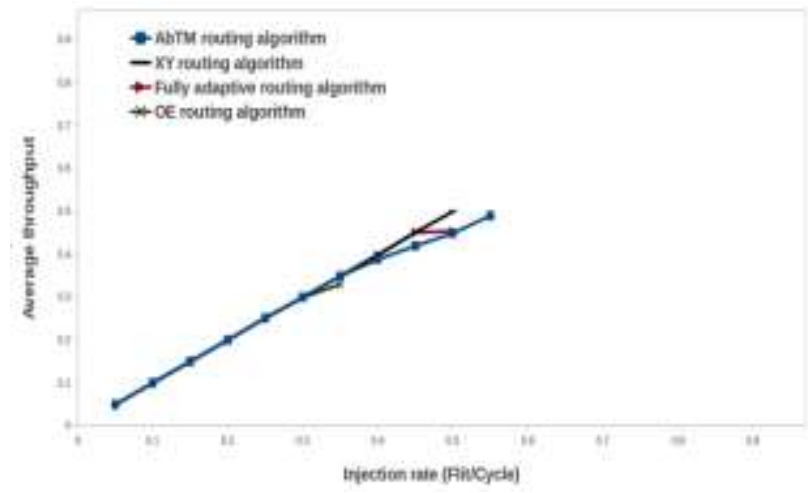

Figure (11) : Average throughput of various routing algorithms on bitcomp traffic pattern.

Figure 12 and Figure 13 demonstrate the latency and throughput for the routing algorithms applied to the shuffle traffic pattern respectively. The worst latency performance is for XY routing algorithm. The network starts to reach saturation using $\mathrm{OE}$ and fully adaptive routing algorithms with the same order while increasing the injection rate. Although AbTM does not show the best latency values in some values of the injection rate, it achieves the highest throughput among all other routing algorithms.

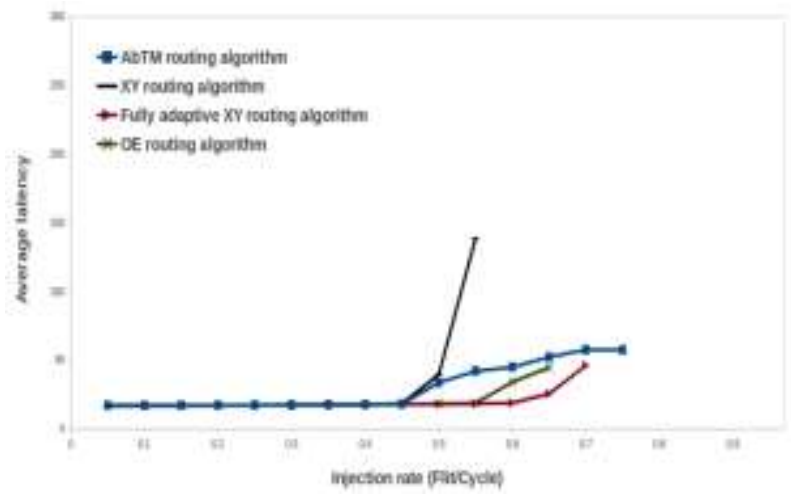

Figure (12): Average latency of various routing algorithms on shuffle traffic pattern

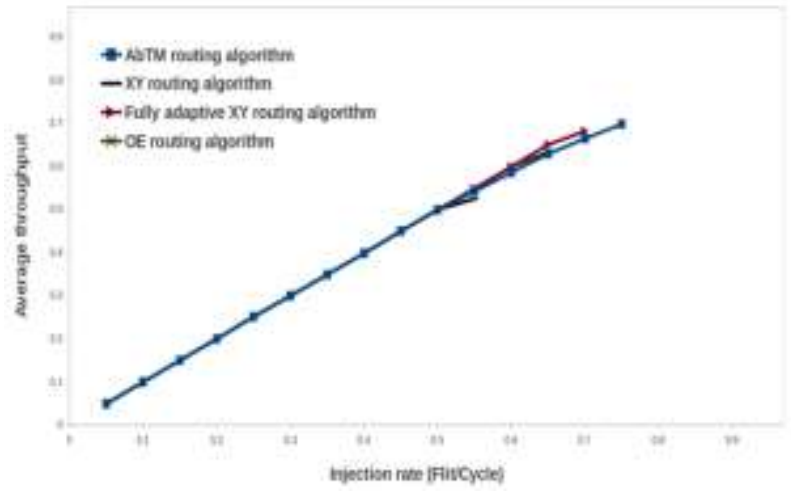

Figure (13) : Average throughput of various routing algorithms on shuffle traffic pattern. 
In general the AbTM proved to achieve the best results in comparison with other routing algorithms as we investigated in the previous figures. Moreover, AbTM results are even better than that obtained in [14] with the same network configurations..

\section{Conclusion:}

In this paper we investigated the four turn model routing algorithms discussed before among different traffic patterns. No results are gathered in saturation state. In uniform traffic pattern the AbTM has the best latency at high injection rates. In bitrev traffic pattern the AbTM routing algorithm has the best latency values. In shuffle and bitcomp traffic patterns the AbtM has the best values of latencies where the other routing algorithms are in saturation state. The throughput of AbTM routing algorithm is always the highest among all routing algorithm which explains why it is functional where the other routing algorithms are in saturation state.

So according to the previous results AbTM routing algorithm proved to be the best choice for NoC systems handle high injection rates and also proved to be functional in all traffic patterns unlike other routing algorithms.

\section{References:}

[1]P. Guerrier and A. Greiner, "A generic architecture for on-chip packet-switched interconnections," Proceedings Design, Automation and Test in Europe Conference and Exhibition 2000 (Cat. No. PR00537), Paris, 2000, pp.250-256. doi: 10.1109/DATE.2000.840047

[2]P. P. Pande, C. Grecu, A. Ivanov and R. Saleh, "High-throughput switch-based interconnect for future SoCs," The 3rd IEEE International Workshop on System-onChip for Real-Time Applications, 2003, pp.304-310.doi: 10.1109/IWSOC.2003.1213053

[3] W. J. Dally and B. Towles, "Route packets, not wires: on-chip interconnection networks," Proceedings of the 38th Design Automation Conference (IEEE Cat. No.01CH37232), 2001, pp. 684-689. doi:10.1109/DAC.2001.156225

[4]S. Kumar et al., "A network on chip architecture and design methodology," Proceedings IEEE Computer Society Annual Symposium on VLSI. New Paradigms for VLSI Systems Design. ISVLSI, Pittsburgh, PA, USA, 2002, pp.105-112. doi: 10.1109/ISVLSI.2002.1016885

[5] W. 1. Dally and B. Towles, Principles and Practices of Interconnection Network,. San Francisco", CA: Morgan Kaufinann, 2004.

[6]J. Duato, "A Necessary and Sufficient Condition for Deadlock-Free Adaptive Routing in Wormhole Networks," International Conference on Parallel Processing Vol. 1, North Carolina State University, NC, USA, 1994, pp.142-149. doi: 
10.1109/ICPP.1994.36

[7] J. Duato, S. Yalamanchili, L. Ni, Interconnection Networks: An Engineering Approach, Morgan Kaufmann, San Francisco, 2002

[8]Z. Wang and Sheng Ma, Libo Huang, Mingche Lai and W. Shi, Networks-onChip:From Implementations to Programming Paradigms. Morgan Kaufmann is an imprint of Elsevier 2015

[9] Booksim2.0 https:1/nocs.stanford.edulcgi-bin/trac.cgilwikil/Resourcest/Booksim

[10] N. Jiang, D. Becker, G. Michelogiannakis, J. Balfour, B. Towles, J. Kim and W. Dally, "ADetailed and Flexible Cycle-Accurate Network-on-Chip Simulator". In the Proceedings of the IEEE International Symposium on Performance Analysis of Systems and Software, Texas, Austin, USA, pp. 86-96, 2013

[11] M. Palesi and M. Daneshtalab, Routing algorithms in networks-on-chip, Springer Science + Business Media, New York, USA,2014

[12] W. Zhang, L. Hou, J. Wang, S. Geng and W. Wu, "Comparison Research between XY and Odd-Even Routing Algorithm of a 2-Dimension 3x3 Mesh Topology Network-on-Chip," WRI Global Congress on Intelligent Systems, Xiamen, 2009, pp. 329-333.doi: 10.1109/GCIS.2009.110

[13] B. Fu, Y. Han, J. Ma, H. Li and X. Li, "An abacus turn model for time/spaceefficient reconfigurable routing," 38th Annual International Symposium on Computer Architecture (ISCA), San Jose, CA, USA, pp. 259-270, 2011

[14] A. Q. Ansari, M. R. Ansari and M. A. Khan, "Performance evaluation of various parameters of Network-on-Chip (NoC) for different topologies," Annual IEEE India Conference (INDICON), New Delhi, India, pp.1-4, 2015. doi: 10.1109/INDICON.2015.744376. 